Revista Oficial del Poder Judicial

ÓRGANO DE INVESTIGACIÓN DE LA CORTE SUPREMA DE JUSTICIA DE LA REPÚBLICA DEL PERÚ

Vol. 11, n. ${ }^{\circ} 13$, enero-junio, 2020, 559-568

ISSN versión impresa: 1997-6682

ISSN versión electrónica: 2663-9130

DOI: https://doi.org/10.35292/ropj.u11i13.64

\title{
Poder Judicial del Perú. $X$ Congreso Nacional de Jueces del Poder Judicial.
}

Lima: Fondo Editorial del Poder Judicial del Perú, 2018, 164 pp.

0

Esta obra contiene las ponencias de los especialistas y los acuerdos asumidos por los jueces representantes de todos los distritos judiciales a nivel nacional, con motivo de la celebración del $\mathrm{X}$ Congreso Nacional de Jueces del Poder Judicial, llevado a cabo en la ciudad de Tacna los días 13, 14 y 15 de julio de 2017. Asimismo, pertenece a la colección Actas Congreso Nacional de Jueces, serie de trabajos elaborados para su difusión a la ciudadanía en respuesta a la trascendente labor desplegada por los jueces participantes en cada congreso realizado, al constituirse en insumos para la toma de decisiones por parte de los órganos de gobierno del Poder Judicial.

Cabe precisar que cada congreso se celebra anualmente desde el año 2007 y -tal como se expuso mediante la Resolución Administrativa n. ${ }^{\circ}$ 237-2007-CE-PJ (2 de octubre de 2007)responde al anhelo compartido entre la población y los jueces de todas las instancias, de habilitar un servicio de administración 


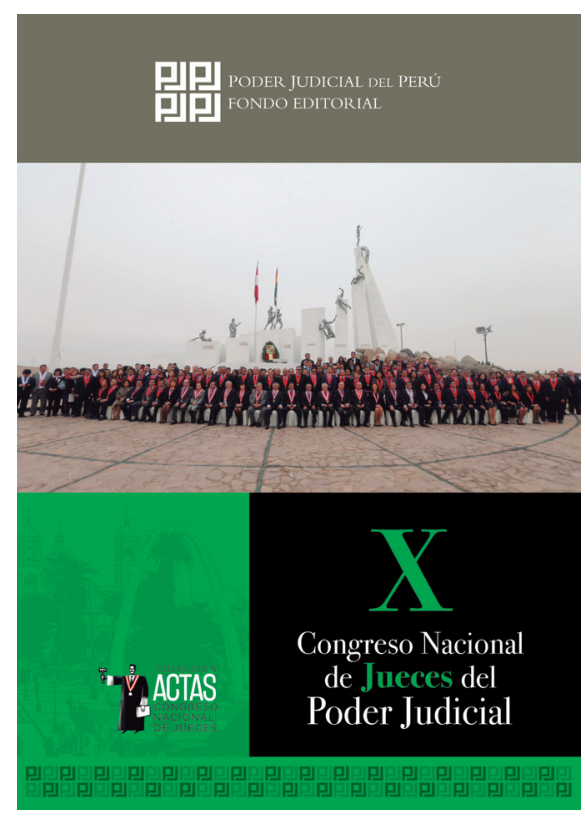

de justicia moderno, eficiente, confiable, predecible y transparente en forma sostenida. En consecuencia, es considerada como una herramienta metodológica para propiciar una reflexión conjunta y con sentido proactivo en búsqueda de la consolidación de las líneas de acción que posibiliten el proceso sostenible de reestructuración del Poder Judicial, así como el análisis de los factores que permitan la concreción de los atributos deseados en la impartición de justicia.

Conforme lo precisa el juez supremo Víctor Prado Saldarriaga en la presentación de la obra en cuestión, los temas recogidos para su elaboración —denominados «ejes temáticos»- fueron inicialmente seleccionados por su Comisión Organizadora y puestos en consideración de los jueces participantes para su discusión en el X Congreso, atendiendo a su amplio debate nacional. En el presente caso, observamos los siguientes: Autonomía y gobierno judicial, Tecnologías de la información, Reformas procesales y despacho judicial, Justicia con perspectiva de género y Lucha contra la corrupción.

En función de dichos ejes temáticos, el libro presenta una estructura dividida en dos partes principales: la primera recoge las disertaciones de los jueces supremos César San Martín Castro, Héctor Lama More, Elvia Barrios Alvarado y Ana María Aranda Rodríguez, así como las de los ponentes Pablo Salvador Coderch (España), José María Morales Arroyo (España), Walter Francisco Gutiérrez Camacho (Perú) y Gian Michael Simmons (Alemania); 
mientras que la segunda detalla las conclusiones de los jueces participantes en el seno del X Congreso.

Las exposiciones de la primera sección nos ofrecen los valiosos aportes que sirvieron de inspiración al momento del debate y la formulación de las conclusiones; por ello, consideramos necesario referir brevemente su contenido.

Sobre el primer eje («Autonomía y gobierno judicial»), encontramos la disertación de Pablo Salvador Coderch, quien explica claramente que el órgano de gobierno del Poder Judicial español es regulado por la Constitución de su país, pese a que esta comprende, dentro de su texto, bastantes posibilidades para que las mayorías políticas del momento introduzcan sus preferencias en cuanto al nombramiento de los doce miembros que le dan mayoría (de un total de veintiuno); el autor expone cómo se pasó de un régimen corporativo a uno parlamentario, luego, a uno asociativo $y$, actualmente, a uno individual o de libre presentación; el autor concluye que prefiere un sistema estable de composición antes que uno que oscile cada cuatro o cinco años.

De otro lado, afirma que la mayor parte de sus jueces y magistrados son de carrera y que su selección básica se efectúa por oposición (con promedio de duración de cinco años y medio) y sin intervención de algún proceso político, de modo que, en términos generales, la justicia española es una justicia íntegra. Respecto al funcionamiento de la justicia en dicho país, manifiesta que están sometidos a una alta carga de trabajo, dada la desproporción existente entre la cantidad de jueces y magistrados frente a la de abogados y población; por tal motivo, la duración de los procesos es un tema preocupante. De igual modo, dentro de su disertación, formula propuestas interesantes como el imprescindible paso del procedimiento escrito a uno oral, así como la reducción de los abanicos salariales, con el objeto de reforzar la independencia, la integridad y la autosatisfacción de los miembros de la carrera judicial. 
Por su parte, José María Morales Arroyo desarrolla y ejemplifica comparativamente los conceptos de separación de poderes, independencia judicial (cuya garantía determina la existencia de la anterior), además de la neutralidad política (entendida como apoliticidad en la toma de decisiones judiciales). Asimismo, grafica situaciones donde los poderes estatales van más allá de los elementos que les son competenciales, rompiendo los criterios de neutralidad y, por tanto, la idea básica de separación de poderes definida constitucionalmente, aconsejando el cumplimiento de la ley y un comportamiento moderado para evitar que las zonas grises nos conduzcan a situaciones de conflictos irresolubles.

En cuanto al segundo eje temático («Tecnologías de la información»), Walter Gutiérrez Camacho nos envuelve en una notable exposición sobre la importancia y los beneficios de la tecnología en el servicio de justicia, por qué actualmente dicho servicio es visto como anacrónico. El autor pronostica cómo, dentro de quince años, la abogacía y la judicatura dejarán de existir tal y como las conocemos debido al empleo de las instancias de gestión de los «jóvenes digitales»; nos aconseja apostar por un cambio de mentalidad, cuando miramos al derecho, e introducir más herramientas tecnológicas en pro de una justicia más eficiente, transparente y con menor corrupción, previa elaboración de un plan de trabajo que proyecte la existencia de una red que genere información para su gestión. Como ejemplo comparado, nos relata los casos Watson, para la medicina, y Ross, para el derecho.

En consonancia con lo anterior, el juez supremo Héctor Lama More refiere cómo el uso de tecnologías en los procesos judiciales y su interoperabilidad es un esfuerzo que realiza el Poder Judicial hace muchos años, integrado dentro de una política no solo del Estado peruano, sino de los Estados a nivel mundial. Dentro de dicho contexto, detalla didácticamente las estrategias utilizadas para la implementación, como piloto, del Expediente Judicial 
Electrónico (instrumento que permite gestionar documentos en los órganos jurisdiccionales a través de medios electrónicos, de modo que evita los inconvenientes asociados a los expedientes físicos, mejora el acceso a la justicia, posibilita una justicia en línea, seguridad en la tramitación de los procesos, transparencia, celeridad y lucha contra la corrupción) inicialmente, en la Corte Superior de Justicia de Lima (con proyección a expandirse en otras Cortes que cuenten con las condiciones requeridas), en las especialidades comercial, tributaria y de mercado, y la Ley Procesal de Trabajo (en la cual se han trabajado productos tecnológicos), además del procedimiento para su utilización en la práctica. Por último, el Dr. Lama More describe los avances en el área de tecnología y sus beneficios.

Acerca del eje denominado "Reformas procesales y despacho judicial», el juez supremo César San Martín Castro nos ilustra sobre el principio de oralidad, sus referencias legales y las notas características del procedimiento oral (el interrogatorio audiovisual, las resoluciones orales, la documentación en el juicio y el proceso electrónico).

En cuanto al eje conocido como «Justicia con perspectiva de género", la jueza suprema Elvia Barrios Alvarado explica, con mucha claridad, por qué hablar de justicia de género constituye un reto, la importancia de la Convención Interamericana para Prevenir, Sancionar y Erradicar la Violencia contra la Mujer y de la Convención sobre la Eliminación de todas las Formas de Discriminación contra la Mujer, el significante de "género» en cuanto proceso de socialización y formación impuesto por la sociedad tanto a hombres como mujeres, la influencia de los estereotipos en el ámbito jurisdiccional y cómo generan muchas desventajas para la mujer y, por último, las acciones que se pueden ejecutar para eliminar las jerarquías de género. 
Con respecto al eje «Lucha contra la corrupción», la jueza suprema Ana María Aranda Rodríguez, entonces jefa del Órgano de Control Interno del Poder Judicial, manifiesta que su objetivo central es la lucha contra la corrupción y describe cómo ha actuado en función de programas, objetivos y estrategias, teniendo como plataforma los instrumentos jurídicos nacionales e internacionales. Considera a la prevención como un pilar fundamental de su actuación y detalla las labores desarrolladas: el acercamiento a las sedes judiciales —inclusive las más lejanas_, la capacitación en temas de derecho y ética, además de las campañas informativas a la población, entre otras. En cuanto a su función correctiva, la ejercen a través de investigaciones disciplinarias y sanciones (desde amonestaciones hasta la propuesta de destitución presentada al entonces Consejo Nacional de la Magistratura). De igual manera, revela cómo se han modernizado los procedimientos internos para el ahorro de costos humanos y materiales, y que se encuentran encaminados al expediente disciplinario digital. Finalmente, estima necesario fortalecer el órgano de control interno y solicita disponer que los magistrados de las oficinas desconcentradas ejerzan dicha función a dedicación exclusiva y que el jefe de cada oficina desconcentrada pertenezca a otro distrito judicial.

Por su lado, Gian Michael Simmons desarrolla de manera muy elocuente la noción de "impunidad», tanto en su dimensión jurídica (el no responsabilizar a un individuo por haber quebrantado el orden jurídico de la sociedad) como esencialmente política, advierte el fracaso del mandato político del Estado al hacer uso del monopolio del poder para mantener el orden social, las relaciones del poder en una comunidad política, y analiza cómo las principales partes interesadas actúan en estas relaciones para hacer valer su poder (lo que incluye hasta la captura del Estado). Asimismo, considera a la «corrupción» como elemento fundamental del lenguaje confrontacional sobre legitimidad 
política, es decir, ética del ejercicio del poder público; suscribe que se constituyen en medios poderosos para la construcción de hechos sociales y en prismas para observar disputas, significados y la estructura de las relaciones sociales. Por último, nos explica las tres formas mediante las cuales «la impunidad de la corrupción» desafía a la sociedad: la primera, referida a la idea de que los miembros de la élite social (actores estatales) pueden cometer actos corruptos sin recibir sanción alguna; la segunda, cuando los actos definidos como criminales permanecen impunes como consecuencia de las relaciones e intereses que definen el funcionamiento de la política y; tercera, la falta de justicia en general. Por ello, promueve su discusión para romper ese círculo vicioso.

La segunda sección de esta obra es aún más valiosa, pues presenta el pensar de los jueces participantes en torno a los ejes temáticos discutidos. Resalta - en primer término- el deseo de los jueces de democratizar y legitimar la elección del presidente del Poder Judicial y de los presidentes de las Cortes Superiores, aspiración que, según comprobamos, fue recogida por el entonces presidente del Poder Judicial, Dr. Víctor Prado Saldarriaga, y remitida al Congreso de la República el 24 de septiembre de 2018, mediante el Proyecto de Ley n. ${ }^{\circ} 3430 / 2018-P J$, Ley que modifica el mecanismo de elección del presidente de la Corte Suprema de la República y de presidentes de las Cortes Superiores de Justicia. Lamentablemente, de acuerdo con el seguimiento que efectuamos en la página web del Congreso de la República, evidenciamos que se encuentra en la Comisión de Constitución y Reglamento desde el 26 de agosto de 2018.

De igual manera, consideramos importante el respaldo al pedido de adjudicación constitucional, para el Poder Judicial, de un porcentaje fijo no menor al $4 \%$ del Presupuesto General de la República. Este pedido no es nuevo y, a pesar de ser necesario 
para asegurar el equilibrio de poderes e independencia judicial, no es escuchado. Advertimos la existencia del Proyecto de Ley n. ${ }^{\circ}$ 1745/2017-PJ, de fecha 4 de agosto de 2017, a través del cual el entonces presidente del Poder Judicial, Dr. Duberlí Rodríguez Tineo, remite al Congreso de la República un conjunto de propuestas, entre las cuales se plantea la reforma constitucional del artículo 145 de la Constitución Política del Perú para la obtención de la asignación presupuestal antes mencionada. Asimismo, en el Proyecto de Presupuesto 2019 Sector 4: Poder Judicial, el Dr. Víctor Prado Saldarriaga, presidente del Poder Judicial, manifestó su respaldo al indicado proyecto de ley al afirmar lo siguiente:

En la fuente Recursos Ordinarios, en los últimos 10 años, la participación del Presupuesto del Poder Judicial en el Presupuesto General de la República ha representado en promedio $2.0 \%$, requiriendo sea una cantidad no inferior al $4.0 \%$ [...], para la creación de más órganos jurisdiccionales, por el aumento de la población y a fin de atender de manera oportuna a la ciudadanía (2018, p. 2).

No obstante, al igual que en el caso anterior, el proyecto está en poder de la Comisión de Constitución y Reglamento desde el 15 de agosto de 2017.

Asimismo, los jueces participantes concluyen que:

el representante de los jueces superiores debe ser elegido con el mismo mecanismo que se aplica para los jueces especializados, por lo que la elección del representante de cada una de las Cortes Superiores de Justicia le debe dar el derecho tanto de elegir por representación como de ser elegido.

Para una mayor ilustración y comprensión del tema, cabría informar a la ciudadanía que la elección de los candidatos de todos 
los distritos judiciales del país para conformar el Consejo Ejecutivo del Poder Judicial se rige por el Reglamento de Elección aprobado por Resolución Administrativa n. ${ }^{\circ}$ 109-94-CE-PJ (10 de noviembre de 1994) y lo establecido en las Resoluciones Administrativas n. ${ }^{\circ} 127-2013-C E-P J$ ( 10 de julio de 2013), n. ${ }^{\circ}$ 123-2015-CE-PJ (25 de marzo de 2015), n. ${ }^{\circ} 159-2015-\mathrm{CE}-\mathrm{PJ}$ (6 de mayo de 2015) y n. ${ }^{\circ}$ 263-2016-CE-PJ (12 de octubre de 2016).

Seguidamente, en cuanto al eje «Tecnologías de la información», optan por una significativa innovación: la eliminación total del papel en las comunicaciones internas y un implemento progresivo del formato electrónico. Dicho pedido tuvo eco con la Resolución Administrativa n. ${ }^{\circ}$ 091-2019-CE-PJ (20 de febrero de 2019), mediante la cual el Consejo Ejecutivo del Poder Judicial aprobó el proyecto Sistema de Gestión Documental (SGD) para el Poder Judicial en pro de la transformación digital del trámite documentario. Esta medida favorecerá la disminución de los costos y la optimización del tiempo empleado en las comunicaciones.

Otra conclusión resaltante gira en torno a la implementación de la oralidad en todos los procesos judiciales no penales. De acuerdo con dicha propuesta, en el año 2018, con el apoyo del Centro de Estudios de Justicia de las Américas (CEJA), se inició la habilitación de la oralidad en los procesos civiles mediante la ejecución de un proyecto piloto en la Corte Superior de Justicia de Arequipa. Ante el éxito de dicha medida (producto de los resultados obtenidos), se está replicando en otras Cortes Superiores como Lima, La Libertad, Ventanilla, Callao, Ica, entre otras.

También llama la atención que los jueces participantes resalten la necesidad de crear un banco de buenas prácticas como medio de difusión de experiencias innovadoras en materia de despacho judicial y que el Poder Judicial peruano haya incorporado en su página web una sección destinada a su difusión, con el objeto de 
que las prácticas premiadas y realizadas por las diferentes Cortes Superiores de Justicia sean aplicadas en los demás distritos judiciales para beneficio de todos los ciudadanos. Ello muestra una sintonía en el trabajo de los jueces y los órganos de gestión del Poder Judicial.

Concluimos reafirmando que esta publicación nos brinda la oportunidad de conocer las inquietudes, las reflexiones y los planes de los miembros de la judicatura nacional respecto a importantes temas del quehacer jurídico; además, nos motiva a meditar y evaluar el proceso de toma de decisiones institucionales para coadyuvar con aportes que permitan alcanzar el ideal de justicia que todos deseamos.

\section{REFERENCIA}

Prado Saldarriaga, V. (2018). Proyecto de Presupuesto - 2019 Sector 4: Poder Judicial. Lima: 28 de noviembre de 2018. Recuperado de http://www.leyes.congreso.gob.pe/Documentos/2016_2021/ Proyectos_de_Ley_y_de_Resoluciones_Legislativas/Anexos/ PODER_JUDICIAL_20181128.pdf

JÉssica Natalí Ramírez CÁRdenas Centro de Investigaciones Judiciales del Poder Judicial (Lima, Perú)

Contacto:jnramirez@pj.gob.pe https://orcid.org/0000-0003-4085-3510 